\title{
Morfometría y Biotipo de Futbolistas Varones Categorías Sub 15 y Sub 16 Pertenecientes a una Escuela Deportiva
}

\author{
Morphometry and Biotype of Male Soccer Players Categories \\ Under 15 and Under 16 from a Sports School
}

Omar Espinoza Navarro'; Jaime Silva Rojas²; Nelson Mallea San Román \& Leonidas Brito Hernández

ESPINOZA, N. O.; SILVA, R. J.; MALLEA, R. N. \& BRITO, H. L. Morfometría y biotipo de futbolistas varones categorías sub 15 y sub 16 pertenecientes a una escuela deportiva. Int. J. Morphol., 39(3):710-715, 2021.

RESUMEN: En las categorías deportivas juveniles, los parámetros morfológicos y biotipológicos son difíciles de determinar ya que es complejo separar los cambios propios de la formación deportiva, de los asociados al crecimiento y la maduración. El objetivo de esta investigación fue establecer características antropométricas y perfiles somatotípicos de adolescentes varones futbolistas chilenos de una escuela deportiva. La muestra estuvo compuesta por 23 jóvenes categoría sub 15 y 30 jóvenes sub 16. Se registraron el peso (kg), estatura $(\mathrm{cm})$, seis pliegues cutáneos, dos diámetros y cinco perímetros. El índice de masa muscular (IMC) se calculó según fórmula de Quetelet. El fraccionamiento de la masa corporal se estimó según la masa grasa (MG), masa muscular (MM), masa residual (MR) y masa ósea (MO).Se describió el somatotipo utilizando el método de Heath \& Carter. Los datos fueron analizados en estadístico para tendencia central, porcentajes y significancia según prueba $\mathrm{t}(\mathrm{p}<0,05)$. Los resultados no muestran diferencias significativas en los patrones antropométricos y biotipológicos entre las dos categorías. Los promedios observados fueron: peso: 60,2 $\pm 7,6$ (kg); Talla: 169,1 $\pm 5,3$ (cm); IMC: 21,2 \pm 2,0; \% MG: 24,3 $\pm 2,7 ; \%$ MM: 45,2 $\pm 2,9$. Somatotipo para jóvenes sub 15 fue de: 2,4 $\pm 0,7 / 4,5 \pm 0,8 / 3,0 \pm 1,1$ y para sub 16 de: $2,3 \pm 1,0 / 4,3 \pm 0,8 / 3,0 \pm 0,9$. Estos resultados son similares a los observados en otras escuelas deportivas de Chile y el mundo. Se concluye que altos porcentajes de masa musculary los componentes meso-ectomórficos, serían características morfológicas en este tipo de deportistas. Se sugiere considerar estos factores, además de considerar los factores sociales y psicológicos, la maduración corporal, centrando el quehacer de los adolescentes más en favorecer un estilo de vida sana y activa que en lo competitivo.

PALABRAS CLAVE: Adolescentes; Biotipología; Masa muscular; Fútbol joven; Vida sana.

\section{INTRODUCCIÓN}

La población adolescente, abarca un período de entre los 10 a los 19 años de edad y se caracteriza por ser una etapa muy compleja que marca la transición de la infancia al estado adulto con cambios biomorfológicos, psicológicos, intelectuales y sociales (Organización Mundial de la Salud, 2014). Las disciplinas deportivas en las categorías juveniles, cada vez se profesionalizan más, poniendo más atención en habilidades de los participantes, sin considerar el lógico desarrollo y el paso a categorías adultas, donde la masa muscular y la fuerza serán las características fundamentales en el rendimiento de la disciplina deportiva profesional (Jorquera Aguilera et al., 2012). Macedo-Uchôa et al. (2019), recomiendan que para un transición adecuada en esta población, conjugar la actividad física regular, con una dieta saludable.
Uno de los deportes mas estudiados en el mundo es el fútbol, existiendo un gran interés en analizar los perfiles morfológicos de los jugadores sin embargo se han detectado varios factores que influyen en el mejor rendimiento, como difererencias significativas entre nacionalidades, categorías y entre las posiciones en el campo de juego. En el fútbol joven estimar los perfiles corporales es más difícil, debido a que es posible observar deportistas con diferentes edades biológicas y cronológicas, debido a la influencia de la etapa puberal y post puberal (Herdy et al., 2015). La selección de jugadores talentosos, deberían incluir pruebas de evaluación de rendimiento físico y motor, la antropometría y la biotipología, además del control del crecimiento y desarrollo de los adolescentes y la correcta ingesta nutricional (Grijota Pérez et al., 2017; Sylejmani et al., 2019).

\footnotetext{
${ }^{1}$ Departamento de Biología, Facultad de Ciencias, Universidad de Tarapacá, Arica-Chile.

${ }^{2}$ Departamento de Nutrición y Dietética, Facultad de Ciencias de la Salud, Universidad de Tarapacá, Arica-Chile.

${ }^{3}$ Programa Magister Motricidad Humana, Universidad de Tarapacá, Arica-Chile.

${ }^{4}$ North American College, Arica-Chile.
} 
Las variables antropométricas ayudan a la selección de los atletas y en los resultados de búsqueda de talentos. Sin embargo, en las categorías básicas, se hace complejo ya que es difícil separar los cambios propios de la formación deportiva, de aquellos asociados con el crecimiento y la maduración durante la adolescencia (Marques de Moraes et al., 2013; Espinoza-Navarro \& Brito-Hernández, 2020). Los objetivos de este estudio fueron establecer las características antropométricas y los perfiles somatotípicos de adolescentes futbolistas chilenos de una escuela de formación deportiva de la ciudad de Arica.

\section{MATERIAL Y MÉTODO}

El diseño de este estudio corresponde a un modelo descriptivo, transversal. La muestra estuvo compuesta por 53 adolescentes varones, 23 jóvenes categoría sub 15 y 30 jóvenes sub 16, integrantes de una Escuela de Futbol Joven (Club San Marcos de Arica), cuyos protocolos de actividad física contemplan una práctica deportiva de 6 horas semanales de entrenamiento (Mallea \& Espinoza-Navarro, 2019). Todos ellos fueron evaluados en el mismo establecimiento deportivo, en horario de la mañana, con ropa ligera y sin zapatos. Se les registró el peso $(\mathrm{kg})$, talla $(\mathrm{cm})$, medición de seis pliegues cutáneos, dos diámetros y cinco perímetros. El índice de masa corporarl (IMC) se calculó según fórmula de Quetelet. El fraccionamiento del peso corporal se determinó de acuerdo a los compartimentos de masa según Ross \& Kerr (1993), se estimaron la masa grasa (MG), masa muscular (MM), masa residual (MR) y masa ósea (MO). Las mediciones se realizaron siguiendo los protocolos de la International Society for Advancement of Kineanthropometry (ISAK). El somatotipo se determinó utilizando el método de Heath \& Carter (Carter, 2002). Los protocolos éticos del proyecto fueron aprobados por el Comité Ético Científico de la Universidad de Tarapacá, posterior a la autorización del Club San Marcos de Arica (julio, 2019). Los datos fueron analizados según descriptiva estadística de tendencia central, desviación estandar y porcentajes. Cuando fue necesario se realizó inferencia utilizando significancia $\mathrm{p}<0,05$ (t-test).

\section{RESULTADOS}

Los resultados antropométricos y biotipológicos de los varones futbolistas adolescentes, se observan en las Tablas I, II, III, IV y en las Figuras 1 y 2, según categoría de clasificacción (sub 15, sub 16).
Tabla I. Características antropométricas de los adolescentes futbolistas varones, categorías sub 15 y sub 16. (Media $\pm \mathrm{DE})$.

\begin{tabular}{lrrrr}
\hline & Sub 15 (n=23) & & Sub 16 (n=30) \\
\hline Variable & & & & \\
Edad (años) & 14,9 & 0,3 & 15,9 & 0,3 \\
Estatura (cm) & 168,7 & 6,1 & 16,5 & 4,6 \\
Peso (kg) & 60,3 & 8,1 & 61,2 & 7,2 \\
& & & & \\
Pliegues(mm) & & & & \\
Biceps & 4,2 & 1,4 & 6,8 & 4,2 \\
Tríceps & 8,7 & 2,4 & 7,7 & 1,9 \\
Subescapular & 7,7 & 2,2 & 7,7 & 2,0 \\
Abdominal & 11,3 & 3,7 & 10,3 & 3,8 \\
Supraspinal & 7,7 & 2,6 & 8,3 & 2,6 \\
Muslo anterior & 10,8 & 4,1 & 10,2 & 4,4 \\
Pantorrilla media & 8,0 & 3,1 & 7,1 & 3,1 \\
Pantorrila máxima & 34,9 & 2,3 & 35,0 & 2,0 \\
& & & & \\
Diámetros (cm) & & & & \\
Humeral & 6,8 & 0,3 & 6,7 & 0,3 \\
Femur & 9,5 & 0,4 & 9,5 & 0,4 \\
& & & & \\
Perímetros (cm) & & & & \\
Brazo superior & 24,0 & 1,4 & 24,0 & 1,4 \\
Brazo superior extendido & 28,0 & 2.0 & 27,7 & 2,1 \\
Cintura & 73,4 & 5,2 & 73,7 & 4,3 \\
Cadera & 91,5 & 5,2 & 91,3 & 4,9 \\
\hline & & & &
\end{tabular}

La Tabla I muestra las características descriptivas de la población de futbol joven sub 15 y sub 16. Según edad (años), estatura (cm), peso $(\mathrm{kg})$. Mediciones de pliegues $(\mathrm{mm})$, diámetros $(\mathrm{cm})$, y perímetros $(\mathrm{cm})$. Media y desviación estandart (Media $\pm \mathrm{DE}$ ).

En la Tabla II se observa la composición corporal de la población en estudio, IMC, medición en porcentajes y en kilógramos de la masa grasa, masa muscular, masa residual y masa ósea. La sumatoria de 6 pliegues cutáneos $\left(\sum\right)$ y somatotipo, según endomorfía, mesomorfía y ectomorfía. (Media \pm DE)

La distribuición según las 13 categorías de somatotipo se observan en la Tabla III. Se muestra que los somatotipo predominantes en ambas poblaciones corresponden a las característivas de mesomorfo balanceado (5) y mesomorfo - ectomorfo (7).

La Tabla IV muestra los promedios y la desviación estándart de las características morfológicas y biotipo de ambas poblaciones en estudio. 
Tabla II. Composición corporal de la población en estudio, IMC, componentes de la masa corporal. Sumatoria de pliegues cutáneos y somatotipo. (Media $\pm \mathrm{DE})$.

\begin{tabular}{lllll}
\hline & \multicolumn{2}{c}{ Sub $15(\mathrm{n}=23)$} & \multicolumn{2}{c}{ Sub $16(\mathrm{n}=30)$} \\
\hline Variable & Media & DS & Media & DS \\
\hline IMC & 21,2 & 2,1 & 21,3 & 1,9 \\
MG $(\%)$ & 24,7 & 2,6 & 23,9 & 2,8 \\
MG $(\mathrm{kg})$ & 14,9 & 2,7 & 14,7 & 2,7 \\
MM $(\%)$ & 44,9 & 3,1 & 45,1 & 2,8 \\
MM (kg) & 27,1 & 4,6 & 27,7 & 3,9 \\
MR (\%) & 11,8 & 0,5 & 12,1 & 0,7 \\
MR (kg) & 7,1 & 1,0 & 7,4 & 1,0 \\
MO $(\%)$ & 12,8 & 1,1 & 12,9 & 1,1 \\
MO (kg) & 7,7 & 0,8 & 7,9 & 0,9 \\
$\sum$ 6 pliegues & 54,2 & 18,1 & 51,3 & 17,8 \\
Endomorfía & 2,4 & 0,7 & 2,3 & 1 \\
Mesomorfía & 4,5 & 0,8 & 4,3 & 0,8 \\
Ectomorfía & 3 & 1,1 & 3 & 0,9
\end{tabular}

MG: masa grasa; MM: masa muscular; MR: masa residual; MO: masa ósea. DE: desviación estándart. $\sum 6$ pliegues: sumatoria de seis pliegues.
Tabla III. Distribución de la población en estudio según los 13 tipos de somatotipos, número y porcentaje.

\begin{tabular}{lll}
\hline Categorías de Somatotipo & $\begin{array}{l}\text { Sub 15 } \\
\mathrm{N}^{\circ} / \%\end{array}$ & $\begin{array}{l}\text { Sub 16 } \\
\mathrm{N}^{\circ} / \%\end{array}$ \\
\hline 1. Endomorfo-balanceado & $0 /(0)$ & $0 /(0)$ \\
2. Endomorfo- mesomórfico & $0 /(0)$ & $1 /(3.3)$ \\
3. Endomorfo-mesomorfo & $0 /(0)$ & $0 /(0)$ \\
4. Mesomorfo-endomórfico & $3 /(13.0)$ & $3 /(10.0)$ \\
5. Mesomorfo balanceado & $11 /(47.8)$ & $9 /(30.0)$ \\
6. Mesomorfo-ectomórfico & $0 /(0)$ & $3 /(10.0)$ \\
7. Ectomorfo-mesomorfo & $6 /(26.1)$ & $9 /(30.0)$ \\
8. Ectomorfo- mesomórfico & $0 /(0)$ & $3 /(10.0)$ \\
9. Ectomorfo balanceado & $2 /(8.7)$ & $0 /(0.0)$ \\
10. Ectomorfo-endomórfico & $0 /(0)$ & $0 /(0.0)$ \\
11. Ectomorfo-endomorfo & $0 /(0)$ & $0 /(0.0)$ \\
12. Endomorfo-ectomórfico & $0 /(0)$ & $0 /(0.0)$ \\
13. Central & $1 /(4.4)$ & $2 /(6.7)$ \\
Totales & $23 / 100$ & $30 / 100$ \\
\hline
\end{tabular}

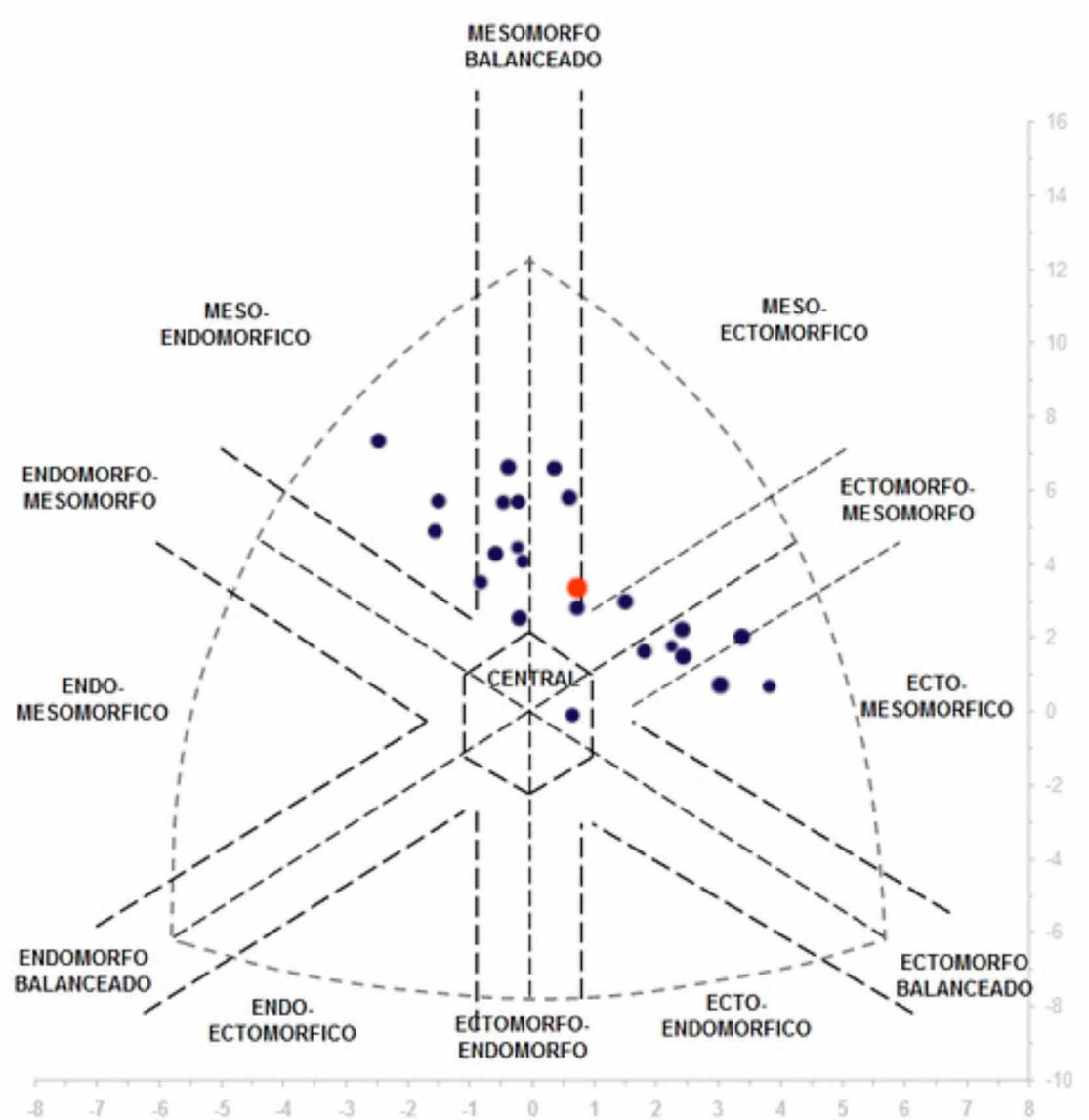

Fig. 1. Somatocarta jóvenes futbolistas sub 15. Valores para mesomorfía, endomorfía y ectomorfía $2,4 \pm 0,7$ / 4,5 $\pm 0,8$ / 3,0 \pm 1, 1 . Punto rojo: promedio somatotipo población 15 años: $4 \pm 0,7 / 4,5 \pm 0,8 / 3,0 \pm 1,1$
La Figura 1 muestra la somatocarta de los jóvenes futbolistassub 15 , con valores de mesomorfía de $2,4 \pm 0,7$, endomorfía de $4,5 \pm 0,8$ y ectomorfía de $3,0 \pm 1,1$.

En la Figura 2 se observa la somatocarta de los jóvenes futbolista sub 16, con valores de 2,3 $\pm 1,0 / 4,3 \pm$ $0,8 / 3,0 \pm 0,9$, para mesomorfía, endomorfía y ectomorfía respectivamente. 
Tabla IV. Promedios y desviación estándart (DE) de las características morfológicas de toda la población en estudio.

\begin{tabular}{lc}
\hline Variable & Media \pm DE \\
\hline Edad (años) & $15.5 \pm 5,7$ \\
Peso (kg) & $60,2 \pm 7,6$ \\
Estatura (cm) & $169,1 \pm 5,3$ \\
Fraccionamiento de masa & \\
1.- Adiposo & \\
MG (\%) & $24,3 \pm 2,7$ \\
MG (kg) & $14,7 \pm 2,7$ \\
2.- Muscular & \\
MM (\%) & $45,2 \pm 2.9$ \\
MM (kg) & $27,4 \pm 4,3$ \\
3.- Residual & \\
MR ( \%) & $12 \pm 0.7$ \\
MR (kg) & $7,3 \pm 1,0$ \\
$4 .-$ Óseo & \\
MO ( \%) & $12,9 \pm 1,0$ \\
MO (kg) & $7,8 \pm 0,9$ \\
IMC (kg/m $\left.{ }^{2}\right)$ & $21,2 \pm 2,0$ \\
$\sum 6$ pliegues & $52,8 \pm 17,5$ \\
\hline
\end{tabular}

MG: masa grasa; MM: masa muscular; MR: masa residual; MO: masa ósea. DE: desviación estándart. $\sum 6$ pliegues: sumatoria de seis pliegues.

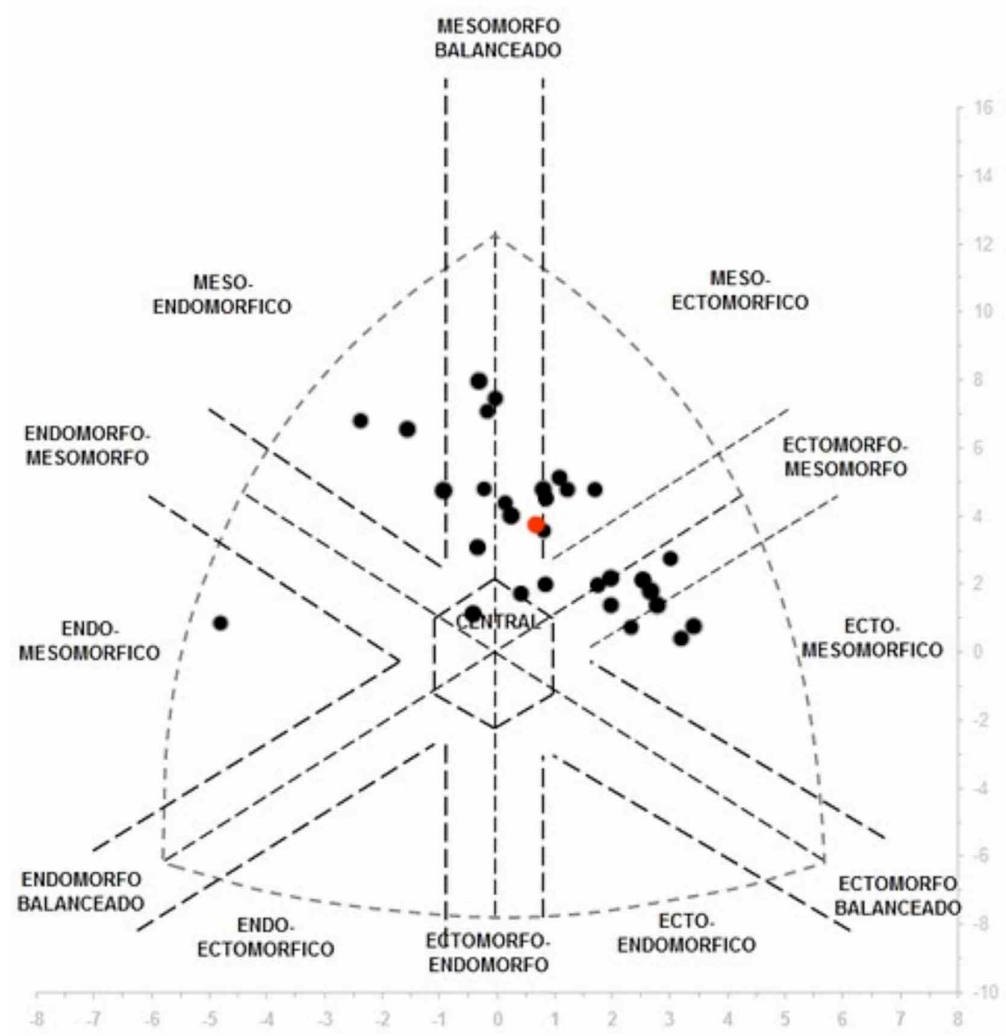

Fig. 2. Somatocarta de los jóvenes futbolistas sub 16 , con valores de $2,3 \pm 1,0$ / $4,3 \pm 0,8 / 3,0 \pm 0,9$, para mesomorfía, endomorfía y ectomorfía respectivamente. Punto rojo: promedio de la población 16 años: $2,3 \pm 1,0 / 4,3 \pm 0,8 / 3,0 \pm 0,9$

\section{DISCUSIÓN}

Los resultados observados en las tablas y figuras, muestran que no existen diferencias significativas entre la morfología y biotipología de los participantes adolescentes entre las categorías sub 15 y sub 16, quienes muestran un fuerte componente mesomórfico. Estos resultados son similares a lo observado por Jorquera Aguilera et al. (2012), quienes también determinan para estos deportistas jóvenes un biotipo con un fuerte componente mesomófico. Herdy et al. también observan una composición corporal semejante en futbolista jóvenes brasileños. Rodríguez Rodríguez et al. (2014), trabajando con deportistas juveniles chilenos de diferentes escuelas deportivas, muestran que la estatura y el fraccionamiento corporal (MG, MM, MR y MO) son similares a los observados en este estudio. Los porcentajes de masa muscular, sobre el $45 \%$, serían características morfológicas propias de esta población y que podrían determinar un mejor rendimiento en este tipo de deportistas juveniles (Godoy-Cumillaf et al., 2015; Curilem Gatica et al., 2016).

Sin embargo, es importante considerar que el rendimiento deportivo es multifactorial y mientras más variables sean controladas durante los entrenamientos y competencias, mayor será la probalidad de resultados exitosos (Godoy-Cumillaf et al.). Las medidas antropométricas, la composición corporal y el somatotipo sirven de insumo a entrenadores, profesores y técnicos, para proponer planificaciones más precisas, para determinar el volumen y la intensidad del entrenamiento, donde además se debe considerar las diferentes posiciones de juego (Herdy et al.; Leão et al., 2019).

Según Rojas Davidson \& Marambio (2017), el prototipo del "nuevo" futbolista chileno ha mejorado en las últimas dos décadas. El biotipo de estos jugadores expresan una condición física destacable y los clubes toman parte en esta evolución. Los complejos deportivos han ido mejorando en cuanto a los implementos en los gimnasios. La industrialización y la globalización del fútbol, están convirtiéndose en factores influyentes para la transformación del nuevo futbolista chileno desde su etapa formativa. La mayoría de los autores coinciden que el paso a categorías superiores y profesionales, supone un nuevo cambio donde el equilibrio 
nutricional será fundamental para alcanzar un aumento en el peso, la estatura y de la masa muscular,características morfológicas que deberían considerarse antes de tomar la decisión de pasar a estas categorías mayores, de lo contrario su carrera deportiva podría ser muy breve (Jorquera Aguilera et al., 2013; Grijota Pérez et al.; Hernández-Jaña et al., 2021).Otros autores de España, Rusia e Italia, muestran la importancia de la maduración somática, los factores sociales, psicológicos y el efecto de la edad relativa en el desarrollo y la transición de estos jóvenes deportistas (Campa et al., 2019; Nikolayecich et al., 2019; Doncaster et al., 2020).

Se concluye que altos porcentajes de masa muscular, bajos porcentajes de masa grasa, mayor estatura y los componentes meso-ectomórficos, serían características morfológicas propias para este tipo de deportistas juveniles. Se sugiere considerar estos factores en la selección de las escuelas deportivas formativas de futbol, además de considerar otras variables, como los factores sociales y psicológicas y la maduración corporal en la proyección de estos jóvenes,centrando el quehacer de los adolescentes más en favorecer un estilo de vida sana y activa que en lo competitivo.

\section{AGRADECIMIENTOS}

Este trabajo contó con el auspicio del proyecto UTA Mayor N 4716-17, Universidad de Tarapacá, Arica-Chile. Los autores declaran que no existen conflictos de intereses.

ESPINOZA, N. O.; SILVA, R. J.; MALLEA, R. N. \& BRITO, H. L. Morphometry and biotype of male soccer players categories under 15 and under 16 from a sports school. Int. J. Morphol., 39(3):710-715, 2021.

SUMMARY: In youth sports categories, morphological and biotypological parameters are difficult to determine since it is complex to separate the changes inherent to sports training from those associated with growth and maturation. The objective of this research was to establish anthropometric characteristics and somatotypic profiles of adolescent Chilean male soccer players from a sports training school. The sample consisted of 23 young people under 15 and 30 young people under 16 . Weight $(\mathrm{kg})$, height $(\mathrm{cm})$, six skin folds, two diameters and five perimeters were recorded. The muscle mass index (BMI) was calculated according to the Quetelet formula. The fractionation of body mass was estimated according to fat mass (FM), muscle mass (MM), residual mass $(\mathrm{RM})$, and bone mass (BM). The somatotype was described using the Heath \& Carter method. The data were analyzed statistically for central tendency, percentages and significance according to the $t$ test $(p<0.05)$. The results show anthropometric and biotypological patterns without significant differences between the two categories.
The averages observed werefor: weight: $60.2 \pm 7.6(\mathrm{~kg})$; Size: 169.1 \pm 5.3 (cm); BMI: $21.2 \pm 2.0$; \% FM: $24.3 \pm 2.7 ; \%$ MM: $45.2 \pm 2.9$; $\%$ RM: $12,0 \pm 0.7 ; \%$ BM: $12,9 \pm 1,0$. Somatotype for youth 15 years was: $2.4 \pm 0.7 / 4.5 \pm 0.8 / 3.0 \pm 1.1$ and for 16 years it was: $2.3 \pm 1.0 / 4.3 \pm 0,8 / 3.0 \pm 0.9$. These results are similar to those observed in other sports schools in Chile and the world. It is concluded that high percentages of muscle mass and mesoectomorphic components would be morphological characteristics in this type of athlete. It is suggested to consider these factors, in addition to considering social and psychological factors, body maturation, focusing the work of adolescents more on promoting a healthy and active lifestyle than on the competitive aspect.

KEY WORDS: Adolescents; Biotypology; Muscle mass; Youth soccer; Healthy living.

\section{REFERENCIAS BIBLIOGRÁFICAS}

Campa, F.; Silva. A. M.; Iannuzzi, V.; Mascherini, G.; Benedetti, L. \& Toselli, S. The role of somatic maturation on bioimpedance patterns and body composition in male elite youth soccer players. Int. J. Environ. Res. Public Health, 16(23):4711, 2019.

Carter, J. E. The Heath-Carter Anthropometric Somatotype. Instruction Manual. San Diego, Department of Exercise and Nutritional Sciences, San Diego State University, 2002.

Curilem Gatica, C.; Almagià Flores, A.; Rodríguez Rodríguez, F.; Farias, T. Y.; Berral de la Rosa, F.; Martínez Salazar, C.; Jorquera Aguilera, C.; Bahamondes Ávila, C.; Soís Urra, P.; Montero, C. C.; et al. Evaluación de la composición corporal en niños y adolescentes: directrices y recomendaciones. Nutr. Hosp., 33(3):734-8, 2016.

Doncaster, G.; Medina, D.; Drobnic, F.; Gómez-Díaz, A. J. \& Unnithan, V. Appreciating factors beyond the physical in talent identification and development: insights from the FC Barcelona sporting model. Front. Sports Act. Living, 2:91, 2020.

Espinoza-Navarro, O. \& Brito-Hernández, L. Morphological patterns associated with metabolic risk factors in the adolescent school population. Int. J. Morphol., 38(6):1645-50, 2020.

Godoy-Cumillaf, A. E. R.; Valdés-Badilla, P. A.; Soler, N. S.; CarmonaLópez, M. I. \& Fernández, J. J. Anthropometric characteristics of adolescents from different sports training schools. Int. J. Morphol., 33(3):1065-70, 2015.

Grijota Pérez, J.; Díaz García, J.; Pérez Quintero, M.: Sánchez, B.; SiquierColl, J. \& Muñoz Marín, D. Análisis Nutricional en Jóvenes Deportistas Practicantes de Fútbol, Tenis y Baloncesto. Kronos, 16(2):1-9, 2017.

Herdy, C. V.; Moreira Nunez, R. A.; Simão Junior, R. F.; Rodríguez Rodríguez F.; Soares Mattos, D.; Ramos, S.; Texeira, R.; Costa e Silva, G. \& Da Silva Novaes, J. Perfil antropométrico, composición corporal y somatotipo de jóvenes futbolistas brasileños de diferentes categorías y posiciones. Educ. Fis. Dep., 34(2):507-24, 2015.

Hernández-Jaña, S.; Jorquera-Aguilera, C.; Almagià-Flores, A. A.; YáñezSepúlveda, R. \& Rodríguez-Rodríguez, F. Body composition and proportionality in Chilean soccer players. Differences between young and champion elite players. Int. J. Morphol., 39(1):252-9, 2021.

Jorquera Aguilera, C.; Rodríguez Rodríguez, F.; Torrealba Vieira, M. I. \& Barraza Gómez, F. Body composition and somatotype of Chilean soccer players sub 16 y sub 17. Int. J. Morphol., 30(1):247-52, 2012.

Jorquera Aguilera. C.; Rodríguez Rodríguez, F.; Torrealba Vieira, M.; Campos Serrano, J.; Leiva, N. G. \& Holway, F. Anthropometric characteristics of Chilean professional football players. Int. J. Morphol., 31(2):609-14, 2013 
Leão, C.; Camões, M.; Clemente, F. M.; Nikolaidis, P. T.; Lima, R.; Bezerra, P.; Rosemann, T. \& Knechtle, B. Anthropometric profile of soccer players as a determinant of position specificity and methodological issues of body composition estimation. Int. J. Environ. Res. Public Health, 16(13):2386, 2019

Macedo-Uchôa, F.; Pinheiro-Lustosa, R.; Cintra-Andrade, J.; NogueiraGodinho, W.; Marques-Aranha, A.; Deana, N. F. \& Alves, N. The influence of physical activity and eating behaviour on body mass index in children and adolescents: A review the literature. Rev. Chil. Nutr., 46(3):343-51, 2019.

Mallea, N. \& Espinoza-Navarro, O. Determinación de la Composición Corporal y Biotipología de los Futbolistas Jóvenes del Club San Marcos de Arica, Categorías Sub 13, 14, 15 y 16. Tesis de Magíster en Ciencias de la Motricidad Humana. Arica, Departamento de Ciencias de la Actividad Física y el Deporte, Universidad de Tarapacá, 2019.

Marques de Moraes, A.; Moreira Gonçalves, E.; de Oliveira Barbeta, V. J. \& Guerra-Júnior. G. Cross-sectional study of the association of body composition and physical fitness with bone status in children and adolescents from 11 to 16 years old. BMC Pediatr., 13:117, 2013.

Organización Mundial de la Salud (OMS). Salud de la Madre, el Recién Nacido, del Niño y del Adolescente. Ginebra, Organización Mundial de la Salud, 2014. Disponible en: https://www.who.int/ maternal_child_adolescent/ topics/adolescense/dev/es/

Rodríguez Rodríguez, F. J.; González Fuenzalida, H. I.; Cordero Ortiz, J. L.; Lagos Nieto, S.; Aguilera Tapia, R. A. \& Barraza Gómez, F. O. Muscle mass estimation and comparison by segment, in young Chilean athletes. Int. J. Morphol., 32(2):703-8, 2014.

Rojas Davidson, F. \& Marambio, L. La Transformación del Fútbol Joven en Chile: El Futbolista del Nuevo Milenio. Santiago de Chile, Escuela de Periodismo Campus Creativo, Universidad Andrés Bello, 2017.

Ross, W. D. \& Kerr, D. A Fraccionamiento de la masa corporal: un nuevo método para utilizar en nutrición clínica y medicina deportiva. Apunts Educ. Fis. Dep., 18:175-87, 1993.

Sylejmani, B.; Maliqi, A.; Gontarev, S.; Haziri, S.; Morina, B.; Durmishaj, E. \& Bajrami, A. Anthropometric characteristics and physical performance of young elite Kosovo soccer players. Int. J. Morphol., 37(4):1429-36, 2019.

\author{
Dirección para correspondencia: \\ Omar Espinoza Navarro \\ Departamento de Biología \\ Facultad de Ciencias \\ Universidad de Tarapacá \\ Arica \\ CHILE
}

\section{E-mail: oespinoz@academicos.uta.cl}

Recibido : 20-02-2021

Aceptado: 03-04-2021 